\title{
一类广义根环的结构
}

\author{
蒋 海 \\ (北京师范大学数学系, 北京 100875)
}

\section{关锤调 广义根环、DJ-环、需等元}

设 $R$ 是结合环,对于 $R$ 的任意元 $a, b$ 定义“o”乘法 $a \circ b-a+b-a b,(R, 0)$ 是一个宫 单位元的半群. 环 $R$ 的 Jacobson ${ }^{\omega N}$ 根记作 $J(R)$, 文中不再重复. 一个环 $R$ 称为广义根环 如果 $(R, \circ)$ 是它的一些子群的并(半群 $S$ 是其子群的并是指对于 $S$ 的任意元素 $a$ 来说, 俰在 $b 、 e \in S$ 使 $\left.a c-c a-a, a b-b a-e, e^{2}-c\right)$. Clark ${ }^{23}$ 证明了: 若环 $R$ 含主算等元 $e$ (即 $e+J(R)$ 是 $R / J(R)$ 的单位元), 则 $R$ 是广义根环当且仅当 $R-e R e+J(R)$ (作为 加群的直和), 其中 $e R e$ 是强正则环. 本文目的是讨论任意环 $R$ 能表成它的子除环与 $J(R)$ 的直和的充要条件, 从而进一步讨论此类广义根环的结构.

设 $A 、 B$ 是环 $R$ 的两个非空子集, 记

$$
A-B-\{a-b \mid a \in A, b \in B\} .
$$

任取 $a \in R$, 令 $G^{(a)}-\{x \in R \mid x a-a x-x\}$, 易见 $G^{(a)}$ 是 $R$ 的子环, $a \in G^{(a)}$ 当且仅 当 $a^{2}-a$, 如果 $a$ 是左(右)拟正则元, 那未 $G^{(a)}-\{0\}$.

定义 如果对于环 $R$ 的任意元素 $a$ 来说, 集合 $a-G^{(0)}$ 中至少含一个拟正则元, 并且 最多含一个双侧非拟正则元, 则称 $R$ 为 $D J-$ 环.

由定义知,如果 $R-J(R)$ 则 $R$ 是 $D J$-环.

命的 1 设 $R$ 是 $D J$-环, $a \in R$, 则 $a$ 是左(右)拟正则元当且仅当 $a$ 是拟正则元.

命题 2 设 $R$ 是 $D J$-环, 则 $R$ 的单侧理想也是 $D J$-环.

命题 3 环 $R$ 含单位元 $1 \neq 0$, 则下列条件等价.

(i) $R$ 是 $D J$-环. (ii) $R$ 中最多含一个双侧非拟正则元. (iii) $R$ 是除环.

定理 1 设 $R$ 是 $D J$-环, $R \neq J(R)$, 则

(i) $R$ 含等元 $e \neq 0$,且 $e-G^{(e)}$ 是除环. (ii) $R$ 是两个加群 $e-G^{(0)}$ 与 $J(R)$ 的 直和. (iii) 设 $L$ 是 $R$ 的单侧理想则 $J(L)-L \cap J(R)$.

证 (i) 由题意 $R$ 含双侧非拟正则元 $a$, 则有 $b \in G^{(0)}, r \in R$ 使

故

$$
(a-b) \circ r-r \circ(a-b)-0 \text {, }
$$

那么 $b-b r-a \circ r \neq 0$. 另一方面, $0=b(a \circ r+b r-b)-b+b^{2} r-b^{2}, b-b^{2}(a-$ $r)$, 是于 $(b-b r)^{2}-(b-b r) b^{2}(a-r)^{2}-b(r \circ a) b(a-r)^{2}-b^{2}(a-r)^{2}-b-b r$.

(ii) 设 $0 \neq e-e^{2} \in R$, 由命题 2 知 $(1-e) R$ 是 $D J$-环. 如果 $(1-e) R$ 含幂等元

1991 05-30 收稿, 1992-02-12 收修改䅻 
$u \neq 0$, 由 $(1-e) R-(1-e) R e+(1-e) R(1-e)$ 知 $u-x+y$, 其中 $x \in(1-e)$ $R e, y \in(1-e) R(1-e)$. 因 $x+y-y x+y^{2}$, 则 $y^{2}-y \neq 0$, 但 $e y-y e-0$, 故 $(e+y)-G^{(e+y)}$ 中有 $e$ 及 $y$, 则必 $e-y$, 此不可能, 所以 $(1-e) R-J((1-e) R) \subseteq$ $J(R)$. 同样 $R(1-e) \subseteq J(R)$. 因为 $e-G^{(e)}-e R e$, 由命题 3 知 $e R e$ 是除环, 故

$$
R-e R e+R(1-e)+(1-e) R-e R e+J(R) \text {. }
$$

(iii) 设 $L$ 是 $R$ 的左理想, 则 $L$ 是 $D J$-环, 故有 $L-J(L)-L \cap J(R)$, 或有 $0 \neq u-$ $u^{2} \in L$ 使 $L-u R u+J(L)$, 由 $R-u R u+J(R)$, 故 $J(L) \subseteq L \cap J(R) \subseteq J(L)$.

定理 2 若环 $R$ 含幂等元 $e \neq 0$, 其子环 $e-G^{(0)}$ 是除环, 且 $R$ 是两个加群 $e-G^{(c)}$ 与 $J(R)$ 的和, 则 $R$ 是 $D J$-环且 $R \neq J(R)$.

证 由 $e-G^{(e)}-e R e$ 且 $R-e R e+J(R)$ 知 $R / J(R)$ 是除环, 任取 $x \in R, x-$ ere $+k(k \in J(R)$, ere $\in e R e)$, 而 $x$ 是拟正则元当且仅当 ere $\neq e$, 故要证 $R$ 是 $D J$-环, 只要考虑 $x-e+k$ 的对应的集合 $x-G^{(x)}$, 设 $t \in G^{(x)} \cap J(R)$, 则 $t x-x t-t, k t e-$ se. 设 $s \in J(R)$ 使 $s \circ k-k \circ s-0$, 即 $0-(s \circ k) x-k v-z e$, 故 $t k-t$, 从而 $0-$ $s(k \circ s)-t$. 任取 $0 \neq a \in G^{(x)}$, 记 $a-e r e+g$, 这里 $g \in J(R)$, $0 \neq e r e \in e R e$, 那未 $x-a-e-e r e+k-g$, 而 $e-e r e \neq e$, 故 $x-G^{(x)}$ 中只有双侧非拟正则元 $x$. 显然 $J(R)-e R(1-e)+(1-e) R e+(1-e) R(1-e)$, 设 $k-a+b+c$, 其中 $a \in e J$ $(R), b \in J(R) e, c \in(1-e) R(1-e)$, 则有 $\hat{c} \in(1-e) R(1-e)$ 使 $c \circ \hat{a}-\hat{c} \circ c-0$. 令 $f-e+a(1-\hat{\imath})+(1-\hat{\imath}) b+(1-\hat{\imath}) b a(1-\hat{\imath})$, 经计算 $x f-f x-f-f^{2} \neq 0$, 易见 $x-f$ 是 $x-G^{(s)}$ 中的一个拟正则元, 所以 $R$ 是 $D J$-环.

由文献 $[3,4]$ 知

推论 任意环 $R$ 的极小单侧理想是 $D J$-环, 几乎拟正则环是 $D J$-环.

定理 3 设 $R$ 是 $D J$-环, $R \neq J(R)$.

(i) $\forall x \in R$, 则 $G^{(x)}-\{0\}$ 或 $G^{(x)}$ 是除环. (ii) 令 $[x]-\left\{y \in R \mid G^{(y)}-G^{(x)} \neq\{0\}\right\}$, 则 $([x], 0)$ 是一个群, 其单位元是 $G^{(s)}$ 的乘法单位元.

证 (i) $\forall x \in R$, 若 $G^{(x)} \neq\{0\}$, 由定理 2 的证明知 $G^{(x)} \cap J(R)-\{0\}$ 且有 $0 \neq f-$ $f^{2} \in G^{(x)}$, 故 $G^{(x)}-f R f$.

(ii) 任取 $y, z \in[x]$, 则 $(y \circ z) \circ f-f \circ(y \circ z)-y \circ z$, 故 $G^{(y \circ z)}-f R f, y \circ z \in[x]$. 由 (i) 知 $f \in[x]$. 设 $x=e+a+b+c$, 其中 $a \in e J(R), b \in J(R) e, c \in(1-e) R(1-e)$, $e^{2}-e \neq 0$, 由定理 2 知 $f-e+a(1-\hat{c})+(1-\hat{c}) b+(1-\hat{c}) b a(1-\hat{c})$. 令 $x^{\prime}-$ $e+a(1-\hat{\imath})^{2}+(1-\hat{\imath})^{2} b+\hat{c} \circ\left[(1-\hat{c}) b a(1-\hat{c})+b a(1-\hat{\imath})^{2}\right]$, 则 $\hat{x}=f \circ x^{\prime} \circ f \epsilon$ $[x]$, 经计算 $x \circ \hat{x}-\hat{x} \circ x-f$, 故 $([x], 0)$ 是群, 其单位元是 $f$.

命题 4 设 $R$ 是 $D J$-环, $R \neq J(R)$. $e$ 及 $\varepsilon$ 是 $R$ 的两个非零幕等元且 $e \neq \varepsilon$. 则 (i) $e R e \cong \varepsilon R \varepsilon$ 且 $e R e \cap \varepsilon R \varepsilon-\{0\}$; (ii) $\varepsilon R e$ 是除环.

设 $R$ 是 $D J$-环, $R \neq J(R)$. 令 $E-\left\{e \in R \mid e^{2}-e \neq 0\right\}$, 对于任意 $u \in E$, 记 $A^{(*)}-$ $u R(1-u), B^{(u)}-(1-u) R u, C^{(u)}-(1-u) R(1-u), H^{(u)}-E-u$.

命预 5 设 $R$ 是 $D J$-环, $R \neq J(R)$. 其他符号同上. (i) 设 $u, v \in E$, 则 $R u \neq R v$ 当 且仅当 $R u \cap R v \subseteq J(R)$; (ii) 令 $W-\{L$ 的 $\{0\}$, 则 $\bigcap_{L \in W} L-\bigcap_{\in \in B} R u-\bigcap_{* \in B} B^{(*)}-B^{(*)} \cap(0: J(R))$ 是 $R$ 的理想, 其中 $(0 . J(R))-$ 
$\{r \in R \mid r J(R)=0\}$.

对 $D J$-环的右理想集 $W_{0}=\{I \neq J(R) \mid I$ 是 $R$ 的右理想 $\}$ 可作同样讨论,且得类似结果.

命预 6 设 $R$ 是 $D J$-环, $R \neq J(R) . E$ 和 $H^{(e)}$ 意义同上,则以下条件等价:

(i) $H^{(e)}$ 是 $J(R)$ 的子环. (ii) $H^{(e)}-e J(R)+J(R) e$.

(iii) $H^{(e)} H^{(e)}-0 . \quad$ (iv) $\forall \varepsilon, u \in E$ 均有 $H^{(\mathrm{s})}-H^{(s)}$.

定理 4 设 $R$ 是环. 则以下条件等价:

(i) $R$ 是 $D J$-环, $R \neq J(R)$ 且有 $e \in E$ 使 $E-e-J(R)$. (ii) $R-e R e+e R(1-$ $e)+(1-e) R e$, 其中 $0 \neq e-e^{2} \in R$, 子环 $e R e$ 是除环, $e R(1-e)+(1-e) R e$ 是 $R$ 的子环.

定理 5 设 $R$ 是 $D J$-环, $R \neq J(R)$, 其他符号同上. 若有 $e \in E$ 使 $E-e-J(R)$, 则 (i) $R-\bigcup_{u \in B} u R u \cup J(R)$; (ii) 对任意 $\varepsilon 、 u \in E$ 都有 $A^{(s)}-A^{(u)}, B^{(u)}-B^{(s)}$.

定理 6 设 $R$ 是 $D J$-环, $R \neq J(R)$. 令 $\Sigma-\{S \mid S$ 是 $R$ 的理想, $S \neq J(R)\}, \Sigma^{\prime}=$ $\{T \subseteq J(R) \mid T$ 是 $R$ 的理想, 存在 $u \in E$ 使 $u J(R) \subseteq T, J(R) u \subseteq T\}$, 则 $\Sigma\left(\Sigma^{\prime}\right)$ 对于集合包 含关系构成格,且 $\Sigma$ 与 $\Sigma^{\prime}$ 格同构.

证 取 $S \in \Sigma$, 有 $u \in E \cap S$ 使 $S-u R u+J(S), J(S)-S \cap J(R)$, 故 $J(S) \in \Sigma^{\prime}$. 取 $S_{i} \in \Sigma$, 有 $e_{i} \in E \cap S_{i}, i-1,2$, 那么 $e_{1} e_{2} \in S_{1} \cap S_{2} \cap E$, 故 $S_{1} \cap S_{2} \in \Sigma$. 显然 $S_{1}+S_{2} \in \Sigma$. 若 $S_{1} \neq S_{2}$, 由 $S_{i}-e_{1} e_{2} R e_{1} e_{2}+J\left(S_{i}\right)(i-1,2)$ 知 $J\left(S_{1}\right) \neq J\left(S_{2}\right)$. 另一方面, 取 $T_{i} \in \Sigma^{\prime}$, 则有 $u_{i} \in E$ 使 $u_{i} J(R) \subseteq T_{i}, J(R) u_{1} \subseteq T_{i}, i-1,2$. 故 $u_{1} u_{2} J(R) \subseteq u_{1} T_{2} \subseteq T_{1} \cap T_{2} \subseteq T_{1}+$ $T_{2}, J(R) u_{1} u_{2} \subseteq T_{1} u_{2} \subseteq T_{1} \cap T_{2} \subseteq T_{1}+T_{2}$. 若 $T \in \Sigma^{\prime}$, 则有 $u \in E$ 使 $u J(R) \subseteq T, J(R) u \subseteq T$. 易见 $S-u R u+T \in \Sigma$ 且 $T-J(S)$. 故 $\varphi: S \longmapsto J(S)$ 是 $\Sigma$ 到 $\Sigma^{\prime}$ 的构同构映射.

\section{参考文献}

11] Jacobson, N., Amer. Math. Soc., Colloq. Publ., 1956, 37.

[2] Clark,W. E., Canad. J. Math., 20 (1968), 88-94.

[3] Jiang Zimes, Kexue Tongbao, 29 (1984), 10: 1284-1287.

[4] Du Xiankun, J. Marh. Research and Exposition, 9 (1989), 2: 200-202, 199. 